\title{
ARTICLE
}

\section{The myeloarchitecture of impulsivity: premature responding in youth is associated with decreased myelination of ventral putamen}

\author{
Camilla L. Nord (D) ${ }^{1,2}$, Seung-Goo Kim (D) ${ }^{1,3}$, Mette Buhl Callesen ${ }^{4}$, Timo L. Kvamme ${ }^{1,4,5}$, Mads Jensen ${ }^{5}$, Mads Uffe Pedersen ${ }^{4}$, \\ Kristine Rømer Thomsen ${ }^{4}$ and Valerie Voon ${ }^{1,6}$
}

Impulsivity has been suggested as a neurocognitive endophenotype conferring risk across a number of neuropsychiatric conditions, including substance and behavioural addictions, eating disorders, and attention deficit/hyperactivity disorder. We used a paradigm with interspecies translation validity (the four-choice serial reaction time task, 4CSRTT) to assess 'waiting' impulsivity in a youth sample ( $N=99$, aged $16-26$ years). We collected magnetization prepared two rapid acquisition gradient echo (MP2RAGE) scans, which enabled us to measure R1, the longitudinal relaxation rate, a parameter closely related to tissue myelin content, as well as quantify grey matter volume. We also assessed inhibitory control (commission errors) on a Go/NoGo task and measured decisional impulsivity (delay discounting) using the Monetary Choice Questionnaire (MCQ). We found R1 of the bilateral ventral putamen was negatively correlated with premature responding, the index of waiting impulsivity on the 4CSRTT. Heightened impulsivity in youth was significantly and specifically associated with lower levels of myelination in the ventral putamen. Impulsivity was not associated with grey matter volume. The association with myelination was specific to waiting impulsivity: R1 was not associated with decisional impulsivity on the MCQ or inhibitory control on the Go/NoGo task. We report that heightened waiting impulsivity, measured as premature responding on the 4CSRTT, is specifically associated with lower levels of ventral putaminal myelination, measured using R1. This may represent a neural signature of vulnerability to diseases associated with excessive impulsivity and demonstrates the added explanatory power of quantifying the mesoscopic organization of the human brain, over and above macroscopic volumetric measurements.

Neuropsychopharmacology (2019) 44:1216-1223; https://doi.org/10.1038/s41386-019-0343-6

\section{INTRODUCTION}

Adolescence and young adulthood is a biologically critical period, marked by increased risk-taking and maturational changes in cortical and subcortical systems that can confer vulnerability to addiction [1]. Studying individual differences in this period is essential to illuminate the natural variance in cognition and neurodevelopment linked to the risk of psychopathology [2]. Here we investigate individual differences in a key dimensional trait enhanced across a number of neuropsychiatric disorders: impulsivity.

Sometimes a decision made in haste is adaptive, helping an animal escape a potential predator or find food without delay; in other cases, it is better to hesitate and decide only after careful deliberation. Decisions made without hesitation can potentiate maladaptive behaviours, such as seeking long-devalued rewards ('compulsivity') or prematurely responding in anticipation of reinforcement ('impulsivity'). Impulsivity is defined as a trait contributing to 'actions that are poorly conceived, prematurely expressed, unduly risky, or inappropriate to the situation, and which often result in undesirable consequences' $[3,4]$. Although most people occasionally act impulsively, excessive impulsivity has a central role in several neuropsychiatric disorders, including attention deficit hyperactivity disorder (ADHD), substance and behavioural addictions, and affective disorders [5].

Its transdiagnostic relevance makes impulsivity a strong candidate for a 'neurocognitive endophenotype': specific cognitive and neural changes that confer vulnerability to a number of psychiatric conditions [3]. This theory is strengthened by findings from human and animal studies that heightened impulsivity is a risk factor for later drug and behavioural addiction [6, 7], and present in biological relatives of drug users [8]. The mechanism of this association is not entirely known, but one proposal is that impulsivity confers a tendency to over-rely on habit learning and this increased habit formation heightens the risk for compulsive drug-taking behaviour $[6,9]$.

Impulsivity is heterogeneous, consisting of multiple subtypes characterized by distinct but overlapping neurobiological mechanisms $[6,10]$. Impulsivity can be broadly subdivided into

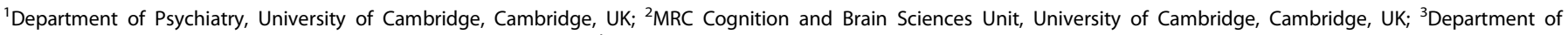

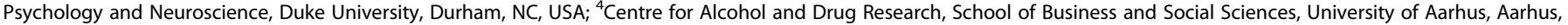

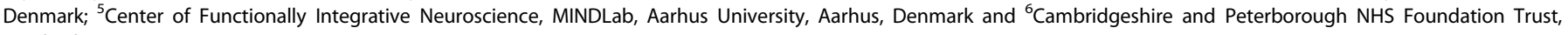
Cambridge, UK

Correspondence: Camilla L. Nord (Camilla.Nord@mrc-cbu.cam.ac.uk)

These authors contributed equally: Camilla L. Nord, Seung-Goo Kim, Kristine Rømer Thomsen and Valerie Voon.

Received: 19 September 2018 Revised: 6 February 2019 Accepted: 7 February 2019

Published online: 15 February 2019 
motoric and decisional forms. Waiting impulsivity or premature responding is a form of motor impulsivity classically measured in animals using the five-choice serial reaction time task (5CSRTT), in which a rodent must respond to brief light cues using nose pokes in one of five apertures [11]. Nose pokes that occur before a light cue (i.e., when the animal does not yet know where to nose poke to receive a reward) are considered premature responses and are punished by a time-out from reinforcement [12]. Rats with a consistent tendency to respond prematurely on the task tend to escalate cocaine self-administration and show other signs of risk for compulsive drug taking [6]. We have recently introduced a human version of the 5CSRTT, the four-choice serial reaction time task (4CSRTT) [12], in which we have shown premature responding (here, releasing the space bar before any cue has been presented) is heightened in a transdiagnostic sample of substance abusers (alcohol- and methamphetamine-dependent patients, cannabis users, and smokers) [12].

Converging human and animal findings suggest impulsivity is subserved by disruptions in limbic and frontrostriatal circuitry [13]. Waiting impulsivity in particular is associated with ventral striatal alterations, with enhanced impulsivity associated with lower ventral striatal D2/3 receptors, conferring an increased risk of transition to habitual cocaine behaviours [14], reduced ventral striatal grey matter density, as well as corresponding reductions in markers of dendritic spines and microtubules [15]. Conversely, ventral striatal lesions attenuate amphetamine-induced increases in premature responding in rats [16]. Similar networks are involved in human premature responding, which is associated with lower resting state functional connectivity of the subthalamic nucleus, ventral striatum, and subgenual cingulate cortex [17], and also associated with increased activity in regions implicated in reward valuation, implying a shift away from higher-level motor preparation and towards compulsivity [18].

A number of studies have revealed associations between impulsivity and grey matter volume (GMV) [19-23]; however, few human studies have focused on the relationship between brain microstructure and impulsivity, despite recent advances in magnetic resonance imaging (MRI) (in one exception, lower mean diffusivity of the putamen, a white matter microstructural measure, was associated with greater impulsivity in past and current methamphetamine users [24]). An important microstructural element of the brain is myelin content: myelin comprises the multilaminar sheaths on axons in the central nervous system formed by oligodendrocytes [25]. Using MRI, maps of relaxation rates can be used to infer myelin content (or myeloarchitecture), an example of in vivo histological techniques that have recently gained popularity in the field [26-28]. These approaches have revealed a relationship between microstructures of the brain (in particular myeloarchitecture) and cognitive functions such as error processing, pitch perception, and cognitive empathy (see ref. [26] for a comprehensive review). In vivo imaging of myeloarchitecture provides insight into local connectomics of the brain, reflecting the density of intracortical connectivity (which could not be described at the 'macroscopic' scale using classical neuroimaging methods) $[26,29,30]$.

Changes in local myelination are thought to be particularly sensitive to brain maturation and plasticity [25]. One recent report found differences in the inferior frontal cortex and insula (measured with myelin-sensitivity magnetization transfer) were associated with a questionnaire measure of impulsivity [31]. However, impulsivity assessed with questionnaire-based measures does not seem to correlate with objective (task-based) measures [32], implying the two may ultimately measure different constructs and limiting the cross-species interpretation of this finding.

A key function of endophenotypes is in predicting vulnerability to a future neuropsychiatric disorder [3]. As such, measuring impulsivity in adolescence and young adulthood, and investigating its relationship with developing microarchitecture could reveal the biology mediating risk for substance and behavioural addictions, ADHD, and other disorders marked by excessive impulsivity, before a disorder is instantiated. In this study, we investigate the relationship between brain microarchitecture and impulsivity in a large youth sample (who completed the 4CSRTT, providing interspecies translational validity to our 'waiting' impulsivity metric). We hypothesized that enhanced waiting impulsivity would be associated with lower ventral striatal GMV and myelination [15] in adolescents and young adults.

\section{MATERIALS AND METHODS}

Participants and procedure

These data are part of a larger study examining risk factors for addictive behaviours in the Danish population (see previous publication for detailed recruitment and full procedure, including measures not reported in this manuscript [33]). We recruited participants in two ways: using a nationally representative survey conducted in 2014 by Statistics Denmark (3064 randomly selected Danes aged 15-25 years) and via local advertisements. The recruited sample included 109 adolescents and young adults (age range 16-26 years), who were selected to have varying levels of externalizing problems (EPs; measured with the YouthMap12 questionnaire [34]), to ensure our sample included participants with an increased risk of problematic substance use [35-37]. Our analysis included 99 participants (those with complete neuroimaging and behavioural datasets) and was made up of participants with no EPs $(N=33)$, minimal EPs $(N=16)$, moderate EPs $(N=21)$, and severe EPs $(N=29)$.

No participants met criteria for a current major psychiatric disorder or criteria for past psychosis or bipolar disorder (as assessed with the Mini International Neuropsychiatric Inventory [38]), and none were currently prescribed psychotropic medication. With the exception of past psychotic and bipolar disorders, only current symptoms were assessed; likewise, we assessed only current use of psychotropic medications. All participants were instructed to abstain from substances for at least $24 \mathrm{~h}$ before study participation (with the exception of tobacco). No participants included in the sample had a history of neurological conditions or severe head trauma.

After the MRI scan, participants completed a computerized version of the 4CSRTT (described below). Data collection took place at the CFIN/MINDLab facilities at Aarhus University, Denmark. The study was approved by the local ethics committee and written consent was obtained before participation. If the participant was under 18, parents also received information about the study to ensure the adolescent's consent was given under parental supervision.

\section{Behavioural measures}

Serial reaction time task. We have previously described the 4CSRTT in detail [12] (see Fig. 1). Briefly, participants were seated in a dimly lit room, wearing headphones for sound attenuation, in front of a touchscreen computer. Participants were instructed to press the space bar with their dominant index finger whenever the cue (four empty boxes) appeared on the screen. After a variable interval $(2-10 \mathrm{~s})$, the target (a green circle) appeared inside one of the boxes, remaining for 32-64 ms. At the appearance of the target, participants released the space bar to touch inside the box where the target had appeared. Throughout, participants were instructed to respond as quickly as possible. The task consisted of four test blocks with monetary feedback (40 trials each) and two baseline blocks without monetary feedback (20 trials each), which were completed before and after the first test block. Baseline block performance (reaction times (RTs) and SDs) was used to adjust monetary feedback amounts on the test blocks: participants won the most money when test RTs were faster than [baseline mean RT $-0.5 \mathrm{SD}$ ] and lost an equivalent 


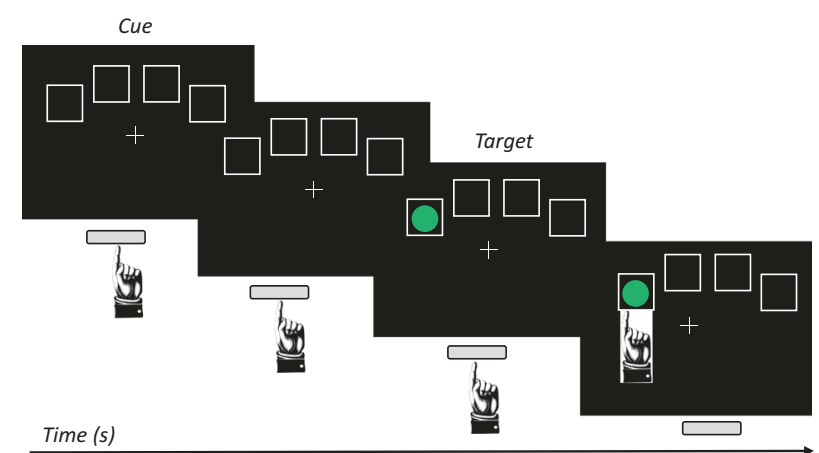

Fig. 1 The four-choice serial reaction time task. Participants responded with their dominant finger on a space bar with the onset of the cue (empty white boxes) and maintained their finger on the space bar until the onset of the target (green circle), at which point they clicked the box displaying the target. Premature responses were defined as early releases of the space bar before the onset of the green circle target

amount when responses were slower than [baseline mean RT + 1.5 SD] (with an intermediate win for RTs equal to [baseline mean $\mathrm{RT} \pm 0.5 \mathrm{SD}$ ], and a small win for those within [baseline mean RT + 1.5 SD]). On trials with premature responses, participants won no money. In test blocks, we also varied the duration and variability of the cue-target interval and introduced a distractor to increase premature responding. The task lasted $20 \mathrm{~min}$ in total.

The primary outcome measure was premature responding, defined as a release of the space bar before the onset of the green circle target.

The task was programmed in Visual Basic with Visual Studio 2005 and Microsoft.NET Framework 2.0 (Microsoft, Redmond, Washington).

Delay discounting task. We also used the Monetary Choice Questionnaire (MCQ) to measure delay discounting, an index of decisional impulsivity [39]. Here, participants chose one of a pair of monetary choices, each associated with a time delay (e.g., 'would you prefer $\$ 10$ today, or $\$ 20$ in 6 months?'). Responses to the questionnaire were then fit to a hyperbolic temporal discounting function by comparing choice preferences with arrays of functions configured to each individual item [40]. This enabled us to quantify discount rate (known as kappa, or k) for each participant. Higher discount rates $(k)$ correspond to more impulsive monetary decision-making on this measure. The MCQ was translated from English to Danish by two Danish researchers with highly proficient English language abilities.

Go/NoGo task. Commission errors were measured using a modified version of the Go/NoGo Task, modified from a previous task [41], which we have described in detail elsewhere [42]. Participants were instructed to press the space bar when 'Go' signals were presented and withhold responding when 'No-Go' signals were presented. The Go/NoGo signals were the letters ' $P$ ' and ' $F$ ' (counterbalanced across participants, such that for some participants ' $P$ ' was the Go signal and ' $F$ ' the NoGo signal and for others ' $F$ ' was the go signal and ' $P$ ' the NoGo signal). The stimuli were displayed randomly but with equal occurrence in one of the four corners of the screen. Following ten practice trials, participants completed the full task. 'Go' signals appeared in $80 \%$ of 400 trials, lasting $\sim 8 \mathrm{~min}$ in total. Trials were presented in a random order. Stimulus duration was $500 \mathrm{~ms}$ and the inter-trial interval was a random interval between 100 and $200 \mathrm{~ms}$ where a central fixation cross was displayed. If a participant missed the $500 \mathrm{~ms}$ deadline, a $300 \mathrm{~ms}$ error tone of $440 \mathrm{~Hz}$ was delivered through the headphones accompanied by a visual feedback text 'you were too slow' presented for $500 \mathrm{~ms}$.
Our primary measure on this task was false alarms, measured as commission errors (participant making a 'Go' response to a 'No Go' signal). Commission errors did not lead to any feedback, except in the initial ten practice trials where a text stimulus ('wrong') was presented after commission errors. The interface was custom programmed in Python using PsychoPy (Version 1.81.0) [43].

Externalizing problems. We employed the YouthMap12 questionnaire [34], which includes six items measuring EPs, developed for the purpose of predicting risk of substance abuse. Participants were asked six questions, e.g., 'to what extent were you/are you and your friends troublemakers?' Participants rated their agreement with these statements and total scores were categorized into one of five possible risk categories. See ref. [34] for a full description of the questionnaire and its development.

Imaging acquisition and processing

A magnetization prepared two rapid acquisition gradient echoes (MP2RAGE) sequence was used to acquire T1 and uniformcontrast images [44]. The sequence was designed to estimate unbiased tissue contrast and also provides an estimate of the longitudinal relaxation time (T1), which is largely influenced by myelin concentration [27, 28]. In line with previous studies, we employed $\mathrm{T} 1$ as a quantitative in vivo marker for grey matter myelination [26-28]. The approach of using R1 maps as a metric of myelin concentration has been previously well-validated; there is strong correspondence between R1 maps and histological postmortem myelin tissue-staining studies [45]. We validated our own sample's anatomical distribution of R1 by comparing it with three previous studies; our R1 data showed a well-known intracortical myelination pattern (i.e., dense intracortical myelination in primary cortices; see Figure S1), as found in previous ex vivo and in vivo data, including MP2RAGE imaging [28, 46, 47]. In addition, a previous cross-sectional study (which included a young sample) found significant increases of myelination in the subcortical regions with age [48]; for this reason, we covaried for age in the main analyses.

Images were acquired by Siemens Skyra 3-T MR system (Erlangen, Germany) with following parameters: TE (time of echo) $=2.98 \mathrm{~ms}$, TI1 (first time of inversion) $=700 \mathrm{~ms}$, TI2 (second time of inversion) $=2500 \mathrm{~ms}$, FA1 (first flip angle) $=4^{\circ}, \mathrm{FA} 2$ (second flip angle) $=5^{\circ}$, TR (time of repetition) $=5000 \mathrm{~ms}$, voxel size $=1 \times 1 \times 1 \mathrm{~mm}^{3}$.

Uniform-contrast (i.e., T1-weighted like) images were segmented and spatially normalized using unified segmentation (30) in SPM12 (7219; https://www.fil.ion.ucl.ac.uk/spm/). We confined our analysis to grey matter by masking the longitudinal relaxation rate images $(\mathrm{R} 1=1 / \mathrm{T} 1)$ with grey matter masks in native space. Next, we nonlinearly transformed the images into Montreal Neurological Institute (MNI) template space using the deformation field resulting from the unified segmentation step, resampled at $1.5 \mathrm{~mm}$ isotropic resolution. We then smoothed them with a $10 \mathrm{~mm}$ full-width at halfmaximum, three-dimensional isotropic Gaussian filter.

In addition to R1 maps, grey matter probability maps were normalized to the MNI template space and modulated by Jacobian determinant of the inverse deformation field to incorporate global brain size variation across individuals, resulting in GMV maps. The GMV maps were resampled and smoothed with the same parameters described above for the R1 maps.

\section{Statistical inference}

To test the association between $\mathrm{R} 1$ and premature responding on the 4CSRTT, we tested the following general linear model (GLM) using Multiple Regression in SPM, including age, sex, and EP as covariates:

$\mathrm{R} 1=\mathrm{PR}+$ Age + Sex + EP $+1+$ Error 
where $P R$ is the premature response, EP is an integer encoding the level of EP as: $1=$ no EPs, $2=$ minimal EPs, $3=$ moderate EPs, $4=$ severe EPs, and error is a zero-mean unit-variance Gaussian error.

The inclusion of the demographic covariates was justified by comparing Model 1 with a reduced model:

$\mathrm{R} 1=\mathrm{PR}+1+$ Error

The F-contrast was computed from Model 1 testing the null hypothesis that the coefficients of age, sex, and EP are all zero.

To test the association while controlling for other types of impulsivity, we further covaried for delay discounting $(k)$ (quantifying decisional impulsivity on the MCQ) and response inhibition, measured as commission errors on the Go/NoGo task:

$$
\mathrm{R} 1=\mathrm{PR}+\mathrm{Age}+\mathrm{Sex}+\mathrm{EP}+k+\mathrm{CE}+1+\text { Error }
$$

where $k$ is the overall delay discount rate on the MCQ and CE is the commisson error rate on the Go/NoGo task.

Whole-brain GLMs were conducted only for the 4CSRTT, in line with our hypotheses about the specific translational relevance of this task for impulsivity, with CE and $k$ rates examined as possible covariates. We defined significance as $p<0.05$, using cluster-level family-wise error rate correction based on random field theory [49], as implemented in SPM12. The cluster forming threshold was $p=0.005$. The cluster extent threshold was determined based on the data as the smallest volume of a cluster with corrected significance below $p=0.05$; this was found to be 2181 voxels $\left(7361 \mathrm{~mm}^{2}\right)$. We restrained the signal space to grey matter to match the processed images.

We used an identical approach to test the association between GMV and premature responding on the 4CSRTT.

\section{Power analysis}

We calculated that we would require 89 participants to have $95 \%$ power to detect a medium effect size of $f^{2}=0.15$ ( $a=0.05$, twotailed; calculated in GPower 3.1.9.2, model: Linear multiple regression); a medium effect size seemed reasonable given previous associations between myeolarchitecture and cognition described in a comparatively smaller sample size [28].

\section{RESULTS}

Demographic and behavioural measures

Descriptive statistics of the demographic and behavioural measures, and their multicollinearity diagnosis scores [50] obtained using MATLAB implementation (https://github.com/brian-lau/ colldiag) are presented in Table 1 . There was a significant correlation between gender and EP $(r=-0.24, p=0.02)$. However, no indication of multicollinearity was found: variance inflation factors (describing the explained variance by all the other variables) of all variables were $<1.2$, well-below a threshold of 5 , and the largest condition index (an index of near-dependency of each eigenvariate) was 8.15 , also well-below a threshold of 30 .

Association between GMV and premature responding

We first tested the association between GMV and premature responding on the 4 CSRTT. There were no significant findings that survived cluster-level random field theory correction (all $p>0.05$ ). Thus, the subsequent results focus on $\mathrm{R} 1$.

\section{Association between $\mathrm{R} 1$ and premature responding}

We first tested for normality of the residual (i.e., noise) distribution of our data using Lilliefors' test; no voxel was found, which significantly deviated from the normal distribution $(\min p=0.15$, false discovery rate adjusted). Therefore, no transformation was applied to the R1 measure. (Data were acquired from unrelated individuals; thus, noise in the data can also be assumed to be independent.) Thus, the data met all assumptions of GLM, allowing us to compare between different models with various terms.

GLM results are presented in Table 2. For Model 1, we found a significant negative correlation between R1 in the left ventral putamen $(T 94]=4.13, p=0.035$, cluster-level corrected) when covarying for age, sex, and EP (Fig. 2a, b). That is, the more an individual made premature responses, the lower the longitudinal relaxation rate (R1) in the left putamen. The set of covariates (age, sex, and EP) as a whole effectively increased explained variance $(F[3,94]=13.17, \quad p=0.0001$, cluster-level corrected) bilaterally across ventral and dorsal striatum and the brain stem (Fig. $2 c$ ).

When controlling for other behavioural measures of inhibitory control (i.e., Model 2, which included $k$, measured with the delay discounting task and commission errors, measured using the Go/NoGo task), we found a negative correlation between $\mathrm{R} 1$ and premature responding in the bilateral ventral putamen (left, $T[92]=4.21, p=0.007 ;$ right, $T[92]=4.07, p=0.010$, cluster-level corrected; Fig. 2 a, b, c). This association was specific to premature responding: there was no association between other measures of inhibitory control ( $k$; commission errors) and R1 in any region, including the bilateral putamina ( $k: p>0.99$; commission errors: $p>0.12$, cluster-level corrected; Fig. $3 \mathrm{~d}-\mathrm{g}$ ).

Exploratory analysis of covariates

We next explored the influence of various sets of covariates on the estimated effect of the premature response and its significance. We tested nested GLMs with covariates of all possible combinations:

$$
\begin{aligned}
& \mathrm{R} 1=\mathrm{PR}+1+\text { Error } \\
& \mathrm{R} 1=\mathrm{PR}+\text { Age }+1+\text { Error } \\
& \mathrm{R} 1=\mathrm{PR}+\text { Sex }+1+\text { Error } \\
& \mathrm{R} 1=\mathrm{PR}+\mathrm{EP}+1+\text { Error }
\end{aligned}
$$

$\mathrm{R} 1=\mathrm{PR}+$ Age + Sex $+1+$ Error

$\mathrm{R} 1=\mathrm{PR}+$ Age $+\mathrm{EP}+1+$ Error

$\mathrm{R} 1=\mathrm{PR}+\mathrm{Age}+\mathrm{Sex}+\mathrm{EP}+k+\mathrm{CE}+1+$ Error

Across all sets of covariates, the effect of premature response was significant $(p<0.0016)$ (see Supplementary Information, Table S1 for full results).

\section{DISCUSSION}

We demonstrate a strong and specific relationship between 'waiting' impulsivity and decreased myelination of the ventral putamen in a relatively large adolescent and young adult population sample. We measured impulsivity using a computer paradigm, which has previously been used to demonstrate heightened premature responding across multiple substance-use dependencies [12], whereas myelin content was quantified using the R1 measure from MP2RAGE anatomical images, which is strongly related to myelin content [26]. These findings reveal a potential myeloarchitectural substrate of premature responding in 
young adulthood: decreased putaminal myelination. In combination with longitudinal work and studies exploring other aspects of impulsivity, this work is a step towards a fuller understanding of how microstructure of the brain contributes to individual differences in impulsive behaviour.

Impulsivity has been suggested as an endophenotype conferring risk for multiple neuropsychiatric disorders, including addictions and $\operatorname{ADHD}[3,5]$. Greater impulsivity, as measured with the human 4CSRTT, the rat 5CSRT, and (human) self-report scales (UPPS-P), is highly associated with addiction-related behaviours $[12,33,51]$. However, impulsivity is multidimensional: the 4CSRTT does not necessarily measure the same construct as the UPPS-P

\begin{tabular}{|c|c|c|c|c|c|c|}
\hline Variables & $\begin{array}{l}\text { Age } \\
\text { [years] }\end{array}$ & $\begin{array}{l}\text { Gender } \\
\text { [female } \\
\text { proportion] }\end{array}$ & EP & PR & k & CE \\
\hline \multicolumn{7}{|c|}{ (1) Descriptive statistics } \\
\hline Mean & 21.69 & 0.30 & 2.51 & 14.71 & 0.009 & 0.49 \\
\hline Std. & 2.69 & - & 1.29 & 12.64 & 0.017 & 0.17 \\
\hline Skewness & 2.69 & - & 0.16 & 12.64 & 0.01 & 0.17 \\
\hline Kurtosis & 2.11 & - & 1.65 & 4.13 & 27.18 & 2.66 \\
\hline \multicolumn{7}{|c|}{ (2) Pearson's correlation ( $p$-value) } \\
\hline Age & & $-0.16(0.12)$ & $\begin{array}{l}0.04 \\
(0.67)\end{array}$ & $\begin{array}{l}0.02 \\
(0.81)\end{array}$ & $\begin{array}{l}-0.12 \\
(0.23)\end{array}$ & $\begin{array}{l}-0.20 \\
(0.05)\end{array}$ \\
\hline Gender & & & $\begin{array}{l}-0.24 \\
(0.02)\end{array}$ & $\begin{array}{l}0.22 \\
(0.31)\end{array}$ & $\begin{array}{l}-0.05 \\
(0.65)\end{array}$ & $\begin{array}{l}0.16 \\
(0.13)\end{array}$ \\
\hline EP & & & & $\begin{array}{l}0.08 \\
(0.41)\end{array}$ & $\begin{array}{l}-0.18 \\
(0.08)\end{array}$ & $\begin{array}{l}-0.17 \\
(0.08)\end{array}$ \\
\hline PR & & & & & $\begin{array}{l}0.04 \\
(0.71)\end{array}$ & $\begin{array}{l}0.18 \\
(0.07)\end{array}$ \\
\hline$k$ & & & & & & $\begin{array}{l}0.03 \\
(0.73)\end{array}$ \\
\hline \multicolumn{7}{|c|}{ (3) Multicollinearity diagnosis scores } \\
\hline VIF & 1.1 & 1.2 & 1.1 & 1.1 & 1.0 & 1.1 \\
\hline
\end{tabular}

$C E$ commission error rate on the Go/NoGo task, EP externalizing problems, $k$ overall delay discount, $P R$ premature response score, VIF variance inflation factor
Impulsive Behaviour Scale, temporal discounting paradigms, or the stop-signal task.

In keeping with this, different measures of impulsivity do not always correlate well with one another and even dissociate in certain situations [5]. This makes it less surprising that the relationship between ventral putaminal myelination and waiting impulsivity was so specific: one might have initially expected a more general association with the related measures we included (i.e., decisional impulsivity, assessed with the MCQ, and inhibitory control, assessed with the Go/NoGo task). However, a key finding from animal and human studies is that impulsivity subtypes are to some degree neurally dissociable $[6,10]$. Therefore, one possibility is that ventral putaminal myelination is only associated with this specific subtype of impulsivity (waiting). Given that impulsivity assessed with questionnaire measures (here, the MCQ) often does not correlate with task-based measures of impulsivity [32], it is also possible that this difference in assessment contributed to the specificity of our finding.

The key facet of impulsivity measured by the 4CSRTT is 'waiting' impulsivity, a construct shown to be crucial to the development of compulsive drug taking in rodents in a seminal paper (although novelty-seeking was essential for the propensity to initiate drug self-administration) [51]. This underlines the importance of measuring 'waiting' impulsivity (with a translational task from the animal literature) in a young population to illuminate neural correlates of risk for substance abuse. Previously, heightened premature responding on the 4CSRTT was associated with blunted functional connectivity between the ventral striatum and bilateral subgenual cingulate cortex (and between the ventral striatum and subthalamic nucleus), consistent with rodent findings using the 5CSRTT $[17,52]$. This network has typically appeared relatively distinct from those regions involved in 'stopping' impulsivity in rodents [53] and humans [17], which tend to be more dorsal.

Our findings were demonstrated using a whole-brain datadriven approach and, critically, are consistent with specific hypotheses from the rodent and human literature [15]. In the context of these findings, our report of decreased ventral putaminal myelination is more dorsal and posterior than the ventral striatum (although our result is located in the ventral putamen). However, few structural (and no microstructural) MRI measures have been associated with premature responding in humans, and volumetric changes in the putamen are very frequently implicated in risk for and current addictive disorders [54-56]. A previous voxel-based morphometry analysis reported lack of premeditation (a cognitive

Table 2. GLM results

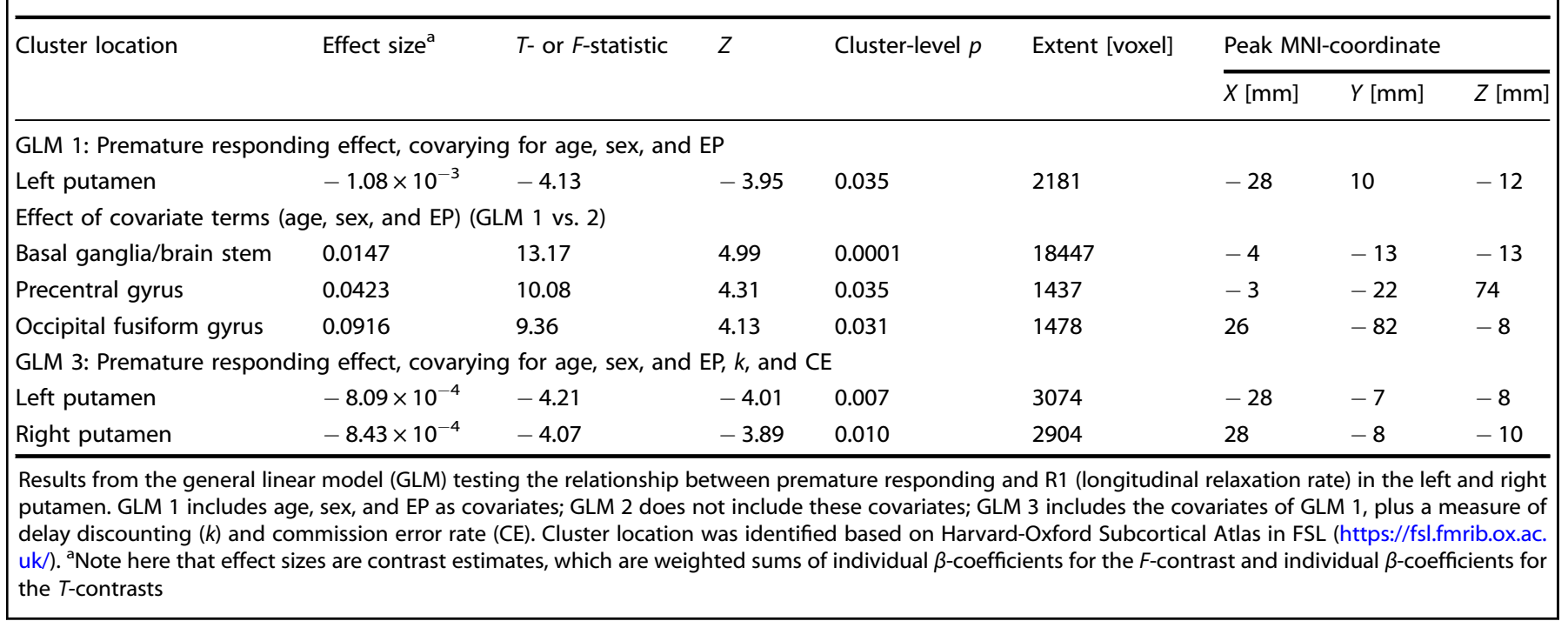



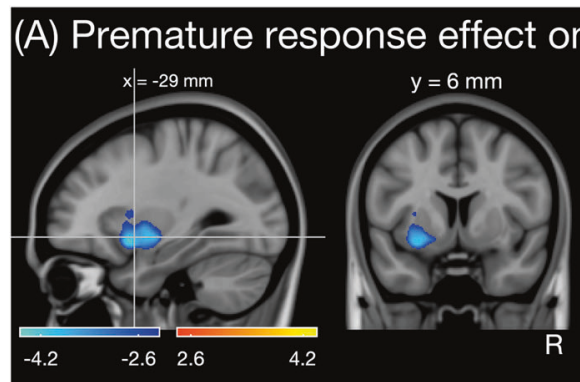

(B) Left putamen

(C) Effect of covariates: GLM(1) vs.GLM(2)
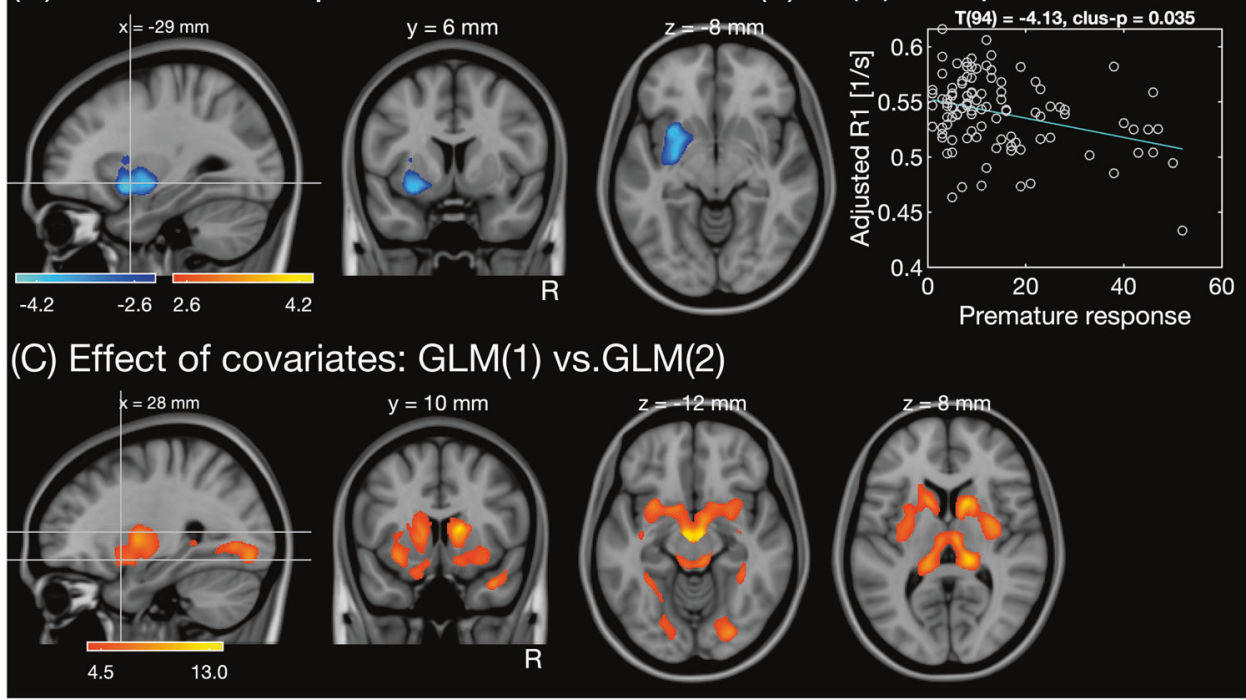

Fig. 2 The relationship between premature responding and longitudinal relaxation rate (R1). a The image shows the regression analysis of premature responding and R1 covaried with age, gender, and externalizing problems. $T$-statistic maps (degrees of freedom $=94$ ) thresholded by height $(|T|>2.63)$ and extent ( $>2180$ voxels), showing voxels surviving cluster-level family-wise error correction $(p<0.05)$. b A scatter plot of R1 adjusted for demographic covariates over premature response scores at the peak voxel in the left putamen is given with a linear regression line. c The image shows the difference between the GLMs of premature responding and R1 with and without covariates of age, gender, and externalizing problems

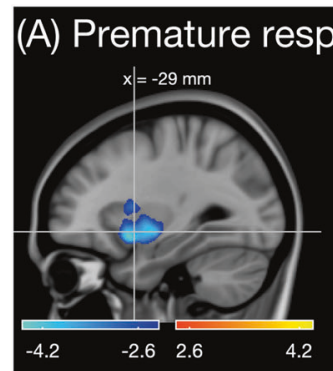

(D) Left putamen

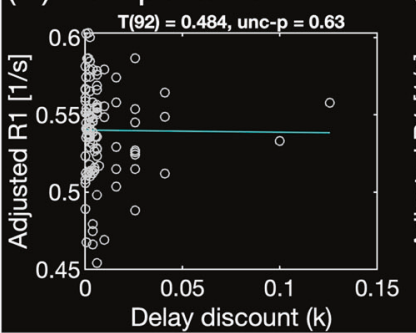

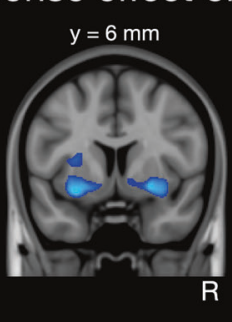

(E) Right putamen

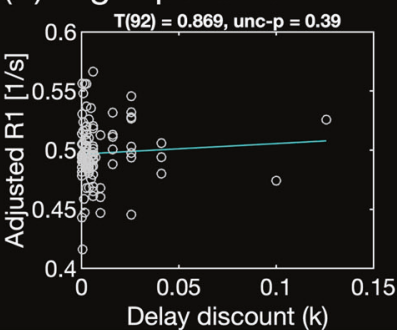

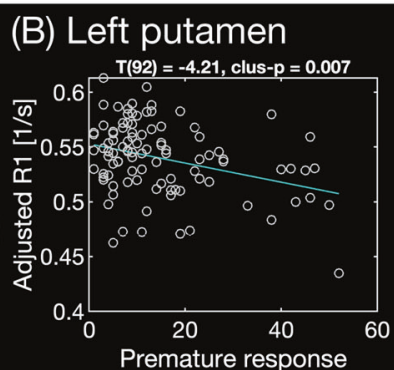

(F) Left putamen

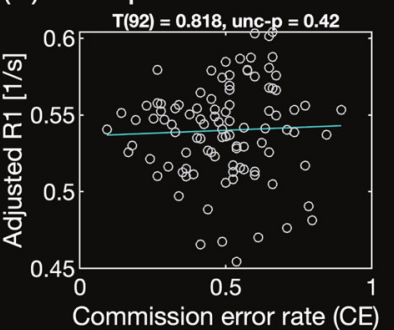

(C) Right putamen

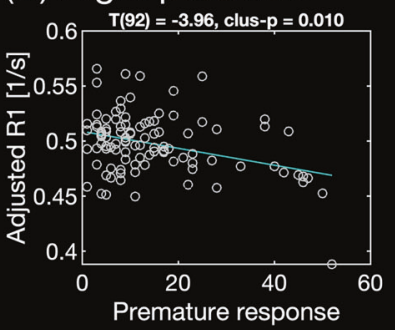

(G) Right putamen

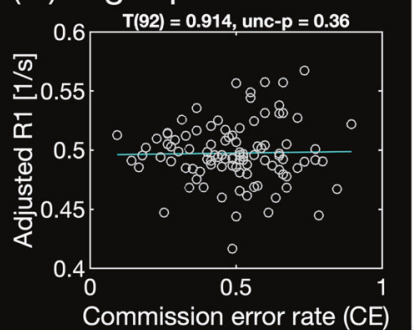

Fig. 3 The relationship between premature responding and longitudinal relaxation rate (R1). a $T$-statistic maps (degrees of freedom $=92$ ) thresholded by height $(|T|>2.63$ ) and extent (> 2903 voxels), showing voxels surviving cluster-level family-wise error correction $(p<0.05)$. b-g Scatter plots of R1 adjusted for covariates over premature response scores at the peak voxel in the left putamen (with a linear regression line)

process related to premature responding) was associated with increased GMV of the putamen in cocaine users [54]; putamen volume itself is $9 \%$ larger in cocaine-dependent individuals [55]. Indeed, an earlier study reported putaminal white matter integrity (decreased mean diffusivity) was associated with heightened impulsivity in both current and past methamphetamine users [24]. These findings support a role for putaminal changes as an endophenotype mediating vulnerability for drug addiction; a key study also reported enlargement of the putamen in both stimulantdependent individuals and their first-degree relatives [56]. Although these studies all investigate macrostructural alterations, increased myelination has been suggested as a likely candidate for decreases in grey matter and increases in white matter observed in adolescent brain development [57]. Therefore, our report of decreased myelination in adolescents and young adults with heightened premature responding could be consistent with previous reports of increased putamen grey matter in adult substance-dependent and at-risk populations [54-56]. We did not observe any relationship between ventral striatal GMV and premature responding. Given the wealth of studies showing associations between cortical and 
subcortical GMV and impulsivity [19-23], this is unexpected; however, this might be a function of our sample age (youth) as well as the relative novelty of our task in humans (4CSRTT). Potentially, grey matter myelination may reflect a more subtle and perhaps earlier biomarker for longer term macrostructural volumetric effects. A longitudinal study is essential to test this possibility.

It is also likely that microstructural changes in the putamen interact with the dopamine system to affect impulsive behaviour. High waiting impulsivity in rodents is characterized by lower ventral striatal D2/3 receptors [14]. The putamen is also a major site of dopamine receptor 2 binding, which is thought to mediate the reinforcing effects of psychostimulants [5]; impulsivity (measured by self-report on the Barett Impulsivity Scale) predicts dopamine receptor binding in the putamen, which is reflective of dopamine transporter availability [58]. Moreover, dopamine has been suggested to have a role in oligodendrocyte differentiation and potentially myelin formation by mature oligodendrocytes [59]. Aberrant dopamine action on oligodendrocytes has even been suggested as a common pathological mechanism across a number of psychiatric disorders, including substance dependence [60]. It is unclear whether myelin abnormalities induce dopaminergic dysfunction, vice versa, or both: although dysfunctional dopamine system activity can affect oligodendrocytes, myelin abnormalities (conferred through genetic risk) may also be a causative factor for dopamine dysfunction [60]. In either case, the mechanism by which disrupted myelination in the ventral putamen during development manifests as heightened premature responding may involve related changes in the dopamine system.

\section{LIMITATIONS}

An important limitation of our finding is our cross-sectional design: a longitudinal study tracking the same adolescents from late childhood through young adulthood could reveal how these myeloarchitectural measures change over time and how that relates to developmental trajectory of impulsive behaviour. However, it is noteworthy that the age we sample at is also a key advantage, as we recruited only people without existing substance-use disorders, some of whom will likely go on to develop one in future. In our sample, family history of psychiatric conditions was not assessed. In future, it will be essential to test whether a family history of substance-use disorders mediates this reported association between heightened waiting impulsivity and lower putaminal myelination in youth. One possibility is that a genetic risk for substance use contributes to myelination differences in the putamen (and other endophenotypic neural changes), behaviourally conferring heightened waiting impulsivity, and increasing risk for substance abuse in future, but this hypothetical relationship should be tested.

In addition, longitudinal relaxation rate is largely, but not exclusively, influenced by myelin content; therefore, other factors affecting longitudinal relaxation rate, such as iron accumulation, or dilation of the perivascular space could have had an (albeit smaller) contribution to our results $[61,62]$. A further possible limitation is that we did not separately assess the potential validity of the Danish version of the MCQ; however, we believe this is unlikely to substantially affect our results, as the nature of the MCQ is such that every question is just a choice between two numerical values (indeed, previous studies have employed Spanish, Dutch, and Bulgarian versions without separate validation, presumably with a similar rationale [63-65]).

Lastly, our participants performed the 4CSRTT outside the scanner; we were therefore unable to measure brain activation associated with premature responding, which would be very useful in future work investigating similar adolescent/young adult samples. Optimally, our work would also be extended to a rodent population, capitalizing on the translational nature of the 4CSRTT/
5CSRTT task and enabling anatomically precise measures of neurotransmitter release to examine the relationship between myelination and dopaminergic activity in the putamen associated with premature responding.

\section{CONCLUSIONS}

The interpretation of our findings could have important implications for understanding the neurodevelopmental processes that confer risk for disorders involving heightened impulsivity. We describe microstructural alterations in the myeloarchitecture of the ventral putamen that are specifically associated with premature responding, a key measure of 'waiting' impulsivity. This provides a putative mechanism describing how structural reorganization in adolescence could confer risk for impulsivity-related disorders via the same circuits implicated in adult humans, as well as rodent models. Our findings emphasize the potential role of microstructural measures as biomarkers of impulsive behaviour in health and disease. The authors declare no competing interests.

\section{FUNDING AND DISCLOSURES}

This work was supported by Aarhus University Research Foundation, Assistant Professor Starting Grant-R46-A4016 (KRT, MBC, VV, MUP), Danish Ministry for Social Affairs and the Interior-9173-0003 (KRT, MBC, MUP), and a Medical Research Council Senior Clinical Fellowship (Grant Number MR/P008747/1 to VV). This work was also supported by NIHR Cambridge Biomedical Research Centre. The authors declare no competing interests.

\section{ADDITIONAL INFORMATION}

Supplementary Information accompanies this paper at (https://doi.org/10.1038/ s41386-019-0343-6).

Publisher's note: Springer Nature remains neutral with regard to jurisdictional claims in published maps and institutional affiliations.

\section{REFERENCES}

1. Chambers RA, Taylor JR, Potenza MN. Developmental neurocircuitry of motivation in adolescence: a critical period of addiction vulnerability. Am J Psychiatry. 2003;160:1041-52.

2. Foulkes L, Blakemore S-J Studying individual differences in human adolescent brain development. Nat Neurosci. 2018;21:315-23.

3. Robbins TW, Gillan CM, Smith DG, de Wit S, Ersche KD. Neurocognitive endophenotypes of impulsivity and compulsivity: towards dimensional psychiatry. Trends Cogn Sci. 2012;16:81-91.

4. Daruna JH, Barnes PA. A neurodevelopmental view of impulsivity. In: McCown WG, Johnson $\mathrm{JL}$, Shure MB, editors. The impulsive client: theory, research, and treatment. Washington, DC, USA: American Psychological Association; 1993. p. 23-37.

5. Dalley JW, Robbins TW. Fractionating impulsivity: neuropsychiatric implications. Nat Rev Neurosci. 2017;18:158.

6. Dalley JW, Everitt BJ, Robbins TW. Impulsivity, compulsivity, and top-down cognitive control. Neuron. 2011;69:680-94.

7. Thomsen KR, Joensson M, Lou HC, Møller A, Gross J, Kringelbach ML, et al. Altered paralimbic interaction in behavioral addiction. Proc Natl Acad Sci USA. 2013;110:4744-9.

8. Ersche KD, Turton AJ, Pradhan S, Bullmore ET, Robbins TW. Drug addiction endophenotypes: impulsive versus sensation-seeking personality traits. Biol Psychiatry. 2010;68:770-3.

9. Everitt BJ, Robbins TW. Neural systems of reinforcement for drug addiction: from actions to habits to compulsion. Nat Neurosci. 2005;8:1481-9.

10. Voon V, Dalley JW. (2015). Translatable and back-translatable measurement of impulsivity and compulsivity: convergent and divergent processes. In: Robbins TW, Sahakian BJ, (eds.) Translational Neuropsychopharmacology. Curr Top Behav Neurosci, vol 28. Springer, Cham.

11. Robbins T. The 5-choice serial reaction time task: behavioural pharmacology and functional neurochemistry. Psychopharmacol (Berl). 2002;163:362-80.

12. Voon V, Irvine MA, Derbyshire $\mathrm{K}$, Worbe $\mathrm{Y}$, Lange I, Abbott $\mathrm{S}$, et al. Measuring "waiting" impulsivity in substance addictions and binge eating disorder in a novel analogue of rodent serial reaction time task. Biol Psychiatry. 2014;75:148-55. 
13. Bari A, Robbins TW. Inhibition and impulsivity: behavioral and neural basis of response control. Prog Neurobiol. 2013;108:44-79.

14. Dalley JW, Fryer TD, Brichard L, Robinson ES, Theobald DE, Lääne K, et al. Nucleus accumbens $\mathrm{D} 2 / 3$ receptors predict trait impulsivity and cocaine reinforcement. Science. 2007;315:1267-70.

15. Caprioli D, Sawiak SJ, Merlo E, Theobald DE, Spoelder M, Jupp B, et al. Gamma aminobutyric acidergic and neuronal structural markers in the nucleus accumbens core underlie trait-like impulsive behavior. Biol Psychiatry. 2014;75:115-23.

16. Cole BJ, Robbins TW. Effects of 6-hydroxydopamine lesions of the nucleus accumbens septi on performance of a 5-choice serial reaction time task in rats: implications for theories of selective attention and arousal. Behav Brain Res. 1989;33:165-79.

17. Morris LS, Kundu P, Baek K, Irvine MA, Mechelmans DJ, Wood J, et al. Jumping the gun: mapping neural correlates of waiting impulsivity and relevance across alcohol misuse. Biol Psychiatry. 2016;79:499-507.

18. Mechelmans DJ, Strelchuk D, Doñamayor N, Banca P, Robbins TW, Baek K, et al. Reward sensitivity and waiting impulsivity: shift towards reward valuation away from action control. Int J Neuropsychopharmacol. 2017;20:971-8.

19. Caravaggio F, Plitman E, Chung JK, Gerretsen P, Kim J, Iwata Y, et al. Trait impulsiveness is related to smaller post-commissural putamen volumes in males but not females. Eur J Neurosci. 2017;46:2253-64.

20. Guo Y, Chen Z, Feng T. The effect of future time perspective on delay discounting is mediated by the gray matter volume of vmPFC. Neuropsychologia. 2017; 102:229-36.

21. Owens MM, Gray JC, Amlung MT, Oshri A, Sweet LH, MacKillop J. Neuroanatomical foundations of delayed reward discounting decision making. Neuroimage. 2017;161:261-70.

22. Tschernegg $M$, Pletzer $B$, Schwartenbeck $P$, Ludersdorfer $P$, Hoffmann U, Kronbichler M. Impulsivity relates to striatal gray matter volumes in humans: evidence from a delay discounting paradigm. Front Hum Neurosci. 2015;9:384.

23. Yip SW, Worhunsky PD, Xu J, Morie KP, Constable RT, Malison RT, et al. Graymatter relationships to diagnostic and transdiagnostic features of drug and behavioral addictions. Addict Biol. 2018;23:394-402.

24. Andres T, Ernst T, Oishi K, Greenstein D, Nakama H, Chang L. Brain microstructure and impulsivity differ between current and past methamphetamine users. J Neuroimmune Pharmacol. 2016;11:531-41.

25. Fields RD. A new mechanism of nervous system plasticity: activity-dependent myelination. Nat Rev Neurosci. 2015;16:756.

26. Edwards LJ, Kirilina E, Mohammadi S, Weiskopf N. Microstructural imaging of human neocortex in vivo. Neuroimage. 2018;182:184-206

27. Stueber C, Morawski M, Schäfer A, Labadie C, Wähnert M, Leuze C, et al. Myelin and iron concentration in the human brain: a quantitative study of MRI contrast. Neuroimage. 2014;93:95-106.

28. Kim S, Knösche TR. Intracortical myelination in musicians with absolute pitch Quantitative morphometry using 7-T MRI. Hum Brain Mapp. 2016;37:3486-501.

29. Amunts K, Zilles K. Architectonic mapping of the human brain beyond Brodmann. Neuron. 2015;88:1086-107.

30. Micheva KD, Wolman D, Mensh BD, Pax E, Buchanan J, Smith SJ, et al. A large fraction of neocortical myelin ensheathes axons of local inhibitory neurons. eLife. 2016;5:e15784.

31. Ziegler G, Hauser T, Moutoussis M, Bullmore ET, Goodyer IM, Fonagy $P$, et al. Compulsivity and impulsivity are linked to distinct aberrant developmental trajectories of fronto-striatal myelination. BioRxiv. 2018:328146.

32. Reynolds B, Ortengren A, Richards JB, de Wit H. Dimensions of impulsive behavior Personality and behavioral measures. Personal Individ Differ. 2006;40:305-15.

33. Rømer Thomsen K, Callesen MB, Hesse M, Kvamme TL, Pedersen MM, Pedersen $\mathrm{MU}$, et al. Impulsivity traits and addiction-related behaviors in youth. J Behav Addict. 2018;7:317-30.

34. Pedersen MU, Thomsen KR, Pedersen MM, Hesse M. Mapping risk factors for substance use: Introducing the YouthMap12. Addict Behav. 2017;65:40-50.

35. Fischer JA, Najman JM, Williams GM, Clavarino AM. Childhood and adolescent psychopathology and subsequent tobacco smoking in young adults: findings from an Australian birth cohort. Addiction. 2012;107:1669-76.

36. Heron J, Barker ED, Joinson C, Lewis G, Hickman M, Munafò M, et al. Childhood conduct disorder trajectories, prior risk factors and cannabis use at age 16: birth cohort study. Addiction. 2013;108:2129-38.

37. Miettunen J, Murray $G$, Jones $P$, Mäki $P$, Ebeling $H$, Taanila $A$, et al. Longitudinal associations between childhood and adulthood externalizing and internalizing psychopathology and adolescent substance use. Psychol Med. 2014;44:1727-38.

38. Lecrubier $\mathrm{Y}$, Sheehan DV, Weiller $\mathrm{E}$, Amorim $\mathrm{P}$, Bonora I, Sheehan $\mathrm{KH}$, et al The Mini International Neuropsychiatric Interview (MINI). A short diagnostic structured interview: reliability and validity according to the CIDI. Eur Psychiatry. 1997;12:224-31.

39. Kirby KN, Petry NM, Bickel WK. Heroin addicts have higher discount rates for delayed rewards than non-drug-using controls. J Exp Psychol Gen. 1999;128:78.
40. Gray JC, Amlung MT, Palmer AA, MacKillop J. Syntax for calculation of discounting indices from the monetary choice questionnaire and probability discounting questionnaire. J Exp Anal Behav. 2016;106:156-63.

41. Houben K, Havermans RC, Nederkoorn C, Jansen A. Beer à No-Go: Learning to stop responding to alcohol cues reduces alcohol intake via reduced affective associations rather than increased response inhibition. Addiction. 2012;107: 1280-7.

42. Kvamme T, Rømer Thomsen $\mathrm{K}$, Callesen M, Donamayor N, Jensen M, Pedersen $M$, et al. Distraction towards contextual alcohol cues and craving are associated with levels of alcohol use among youth. BMC Psychiatry. 2018;18:354

43. Peirce JW. PsychoPy—-psychophysics software in Python. J Neurosci Methods. 2007;162:8-13.

44. Marques JP, Kober T, Krueger G, van der Zwaag W, Van de Moortele P-F, Gruetter R. MP2RAGE, a self bias-field corrected sequence for improved segmentation and T1-mapping at high field. Neuroimage. 2010;49:1271-81.

45. Lutti A, Dick F, Sereno MI, Weiskopf N. Using high-resolution quantitative mapping of R1 as an index of cortical myelination. Neuroimage. 2014;93:176-88.

46. Marques JP, Khabipova D, Gruetter R. Studying cyto and myeloarchitecture of the human cortex at ultra-high field with quantitative imaging: $R 1, R 2^{*}$ and magnetic susceptibility. Neuroimage. 2017;147:152-63.

47. Glasser MF, Coalson TS, Robinson EC, Hacker CD, Harwell J, Yacoub E, et al. A multi-modal parcellation of human cerebral cortex. Nature. 2016;536:171-8.

48. Keuken M, Bazin P-L, Backhouse K, Beekhuizen S, Himmer L, Kandola A, et al. Effects of aging on $\mathrm{T}_{1}, \mathrm{~T}^{*}$, and QSM MRI values in the subcortex. Brain Struct Funct. 2017;222:2487-505.

49. Worsley KJ, Evans AC, Marrett S, Neelin P. A three-dimensional statistical analysis for CBF activation studies in human brain. J Cereb Blood Flow Metab. 1992;12:900-18.

50. Belsley DA, Kuh E, Welsch RE. (2004). Regression diagnostics: identifying influential data and sources of collinearity. Vol. 571. John Wiley \& Sons, Hoboken New Jersey.

51. Belin D, Mar AC, Dalley JW, Robbins TW, Everitt BJ. High impulsivity predicts the switch to compulsive cocaine-taking. Science. 2008;320:1352-5.

52. Jupp B, Caprioli D, Saigal N, Reverte I, Shrestha S, Cumming P, et al. Dopaminergic and GABA-ergic markers of impulsivity in rats: evidence for anatomical localisation in ventral striatum and prefrontal cortex. Eur J Neurosci. 2013;37:1519-28.

53. Eagle DM, Baunez C, Hutcheson DM, Lehmann O, Shah AP, Robbins TW. Stopsignal reaction-time task performance: role of prefrontal cortex and subthalamic nucleus. Cereb Cortex. 2007;18:178-88.

54. Moreno-López L, Catena A, Fernández-Serrano MJ, Delgado-Rico E, Stamatakis EA, Pérez-García M, et al. Trait impulsivity and prefrontal gray matter reductions in cocaine dependent individuals. Drug Alcohol Depend. 2012;125:208-14.

55. Jacobsen LK, Giedd JN, Gottschalk C, Kosten TR, Krystal JH. Quantitative morphology of the caudate and putamen in patients with cocaine dependence. Am J Psychiatry. 2001;158:486-9.

56. Ersche KD, Jones PS, Williams GB, Turton AJ, Robbins TW, Bullmore ET. Abnormal brain structure implicated in stimulant drug addiction. Science. 2012; 335:601-4.

57. Lebel C, Walker L, Leemans A, Phillips L, Beaulieu C. Microstructural maturation of the human brain from childhood to adulthood. Neuroimage. 2008;40:1044-55.

58. Costa A, la Fougère $C$, Pogarell $O$, Möller H-J, Riedel M, Ettinger U. Impulsivity is related to striatal dopamine transporter availability in healthy males. Psychiatry Res Neuroimaging. 2013;211:251-6.

59. Bongarzone ER, Howard SG, Schonmann V, Campagnoni AT. Identification of the dopamine D3 receptor in oligodendrocyte precursors: potential role in regulating differentiation and myelin formation. J Neurosci. 1998;18:5344-53.

60. Feng Y. Convergence and divergence in the etiology of myelin impairment in psychiatric disorders and drug addiction. Neurochem Res. 2008;33:1940-9.

61. Desmond KL, Al-Ebraheem A, Janik R, Oakden W, Kwiecien JM, Dabrowski W, et al. Differences in iron and manganese concentration may confound the measurement of myelin from R1 and R2 relaxation rates in studies of dysmyelination. NMR Biomed. 2016;29:985-98

62. Okubo G, Okada T, Yamamoto A, Fushimi Y, Okada T, Murata K, et al. Relationship between aging and $\mathrm{T} 1$ relaxation time in deep gray matter: $\mathrm{A}$ voxel-based analysis. J Magn Reson Imaging. 2017;46:724-31.

63. Perales JC, Verdejo-García A, Moya M, Lozano Ó, Pérez-García M. Bright and dark sides of impulsivity: performance of women with high and low trait impulsivity on neuropsychological tasks. J Clin Exp Neuropsychol. 2009;31:927-44.

64. Stevens L, Verdejo-García A, Roeyers $H$, Goudriaan AE, Vanderplasschen W. Delay discounting, treatment motivation and treatment retention among substance-dependent individuals attending an in inpatient detoxification program. J Subst Abus Treat. 2015;49:58-64.

65. Vassileva J, Georgiev S, Martin E, Gonzalez R, Segala L. Psychopathic heroin addicts are not uniformly impaired across neurocognitive domains of impulsivity. Drug Alcohol Depend. 2011;114:194-200. 